Chronic Obstructive Pulmonary Diseases: Journal of the COPD Foundation

\author{
Editorial
}

\title{
Forced Expiratory Volume in One Second and Patient-Reported Outcomes: Closer Than You Think
}

\author{
James F. Donohue, $\mathrm{MD}^{1}$
}

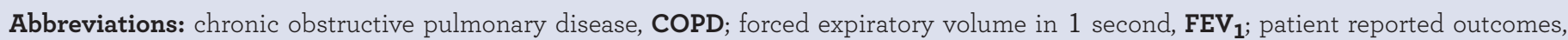
PROs; St. George's Respiratory Questionaire, SGRQ; transition dyspnea index, TDI

Citation: Donohue JF. Editorial: Forced expiratory volume in 1 second and patient-reported outcomes: closer than you think. Chronic Obstr Pulm Dis (Miami). 2016; 3(2):512-514. doi: http://dx.doi.org/10.15326/jcopdf.3.2.2016.0144
\end{abstract}

\section{Pulmonary Diseases and Critical Care, Department of Medicine, University of North Carolina, Chapel Hill}

\section{Address correspondence to:}

James F. Donohue, MD

University of North Carolina

734 West Barbee Chapel Rd

Chapel Hill, NC 27517

email: jdonohue@med.unc.edu

\section{Keywords:}

forced expiratory volume in 1 second; $\mathrm{FEV}_{1}$; patient reported outcomes

Current guidelines for chronic obstructive pulmonary disease (COPD) now recommend that lung function as measured by forced expiratory volume in 1 second ( $\mathrm{FEV}_{1}$ ) be measured alongside patient-reported outcomes (PRO) in clinical trials of inhaled bronchodilators. Important elements of COPD management are based on symptoms, severity, activity limitations or health status. All are relevant to the approval process and clinical use. Future risk of disease progression, particularly exacerbations, must also be considered for management of stable COPD and for consideration by policy makers and payers.

The meta-analysis in this issue of Chronic Obstructive Pulmonary Diseases: Journal of the COPD Foundation by de la Loge and colleagues ${ }^{1}$ explores the relationship between trough $F E V_{1}, F E V_{1}$ area under the curve and PROs including St. George's Respiratory Questionnaire total score (SGRQ), transition dyspnea index (TDI) and exacerbation rates.

In this analysis, the quantity of 24 weeks durationrandom controlled trials was 52 and the number of participants over 62,000; an impressive undertaking. The findings include a large negative correlation $\left(R_{1}\right.$ 0.68 ) between trough $F_{1} V_{1}$ and $S G R Q$. Improvement of $100 \mathrm{ml}$ in $\mathrm{FEV}_{1}$ corresponded to a 5.9 point reduction in SGRQ. The weighted correlation coefficient of trough FEV 1 with TDI, exacerbations rate (all), and with moderate to severe exacerbations are also all $p>0.05$. The conclusion is that a strong association exists between changes in spirometric measurements and changes in PROs.

But historically, while relationships exist at the clinical trial level, at the individual level predictions of COPD outcomes based on trough $\mathrm{FEV}_{1}$ are not possible for a given patient due to the substantial between and within patient variability of these end points. This is evidenced in some studies by the limited magnitude of correlation coefficients despite significant $p$ values.

What can physicians, policy makers, payers and patients take from these and other studies using large databases and meta-analysis? If $\mathrm{FEV}_{1}$ is the primary outcome in pivotal clinical trials of bronchodilators, can we infer from a robust change in $\mathrm{FEV}_{1}$ that exacerbation rates and hospitalization rates will decline while healthrelated quality of life and symptoms will improve?

The answer is still no; at least not yet. The authors and others clearly point out that a major limitation is that individual patient data is unavailable and these meta-analyses are conducted on study level data. In the future, hopefully the individual data will be available for 
large clinical trials and in the public domain. Another limitation of all of these studies is that not all include all the end points of interest.

Other groups have correlated change in lung function with patient outcomes in COPD. In a pooled analysis from 3 studies of 3313 patients, TDI and change in SGRQ improved at all time points with increasing positive change in $\mathrm{FEV}_{1}{ }^{2}$ Also, exacerbation rates over the study duration declined, $p>0.01$. However, individual level correlations were 0.03-0.18 while cohort level correlations were 0.79-0.95. At 28 weeks a $100 \mathrm{ml}$ increase in $\mathrm{FEV}_{1}$ was associated with improved TDI (0.46), change in SGRQ (1.3-1.9) and a $12 \%$ decrease in exacerbation rate. Adjustments for baseline covariant seem to have little impact on the relationship between delta $\mathrm{FEV}_{1}$ and outcomes.

More recently, pooled longitudinal data from 23 randomized studies $(n=23,313)$ explores the relationship of these endpoints using correlations of data summaries and model-based analysis: generalized linear mixed effects regression modelling to determine if change in $\mathrm{FEV}_{1}$ could predict patient outcomes with different treatments. ${ }^{3}$ One intriguing finding was that no evidence of a plateau effect was observed such that patients with the greatest improvement in trough $\mathrm{FEV}_{1}$ had better SGRQs and TDI scores, fewer exacerbations and used less rescue medication. The combination of long-acting beta-agonists/long-acting muscarinicantagonists produced robust changes in $\mathrm{FEV}_{1}$ and had greater effects on the PROs than mono therapies. Does this imply that maximizing lung function will lead to greater PROs? This question remains to be answered by randomized prospective studies.

The relationship of change in lung function, SGRQ and economically significant outcomes of exacerbation and health resource utilization are equally of interest. ${ }^{4}$ These results and those of de la Loge offer providers and players a broader picture of the relationship between $\mathrm{FEV}_{1}$ to inform clinical and formulary decisions while stimulating new research questions for future prospective studies. 


\section{References}

1. de la Loge C, Tugaut B, Fofana F, et al. Relationship between forced expiratory volume in 1 second and patient-reported outcome changes: results of a meta-analysis of randomized trials in stable copd. Chronic Obstr Pulm Dis (Miami). 2016;3(2): TBD doi: http://dx.doi.org/10.15326/jcopdf.3.2.2016.052

2. Jones PW, Donohue JF, Nedelman J, et al. Correlating changes in lung function with patient outcomes in chronic obstructive lung disease : a pooled trial. Respir Res. 2011; 12:161. doi: http://dx.doi.org/10.1186/1465-9921-12-161

3. Donohue JF, Jones PW, Bartels C, et al .Relationship between changes in trough FEV 1 and COPD patient outcomes; Pooled analysis of 23 trials in COPD. Eur Respir J. 2015; 46 (Suppl59).

4. Martin AL, Marvel J, Fahrbach $\mathrm{K}$ et al. The association of lung function and St. George's respiratory questionnaire with exacerbations in COPD: a systematic literature review and regression analysis. Respir Res. 2016;17:40.

doi: http://dx.doi.org/10.1186/s12931-016-0356-1 